\title{
Die Telefonhilfe 147: Immer und überall da für ratlose, ratsuchende und misshandelte Kinder und Jugendliche
}

\author{
M. Wegener
}

Tagtäglich empfangen Sie in Ihrer Praxis Kinder mit alltäglichen Krankheiten. Häufig ist es aber darüber hinaus der (Kinder-)Arzt, der als erster weitergehende Symptome feststellt. Symptome, die auf andere Hintergründe schliessen lassen. Symptome, die die Folge von Misshandlung sein könnten. Wie kann und wie wird diesen Kindern und Jugendlichen zusätzlich zur direkten medizinischen Behandlung geholfen? Wo erhalten diese Kinder und Jugendlichen Vertrauen, Unterstützung und Verständnis? Wo erhalten sie ein offenes Ohr und die professionelle Beratung, die ihnen hilft, aus ihrer Situation aus- und aufzubrechen?

Seit $3 \frac{1}{2}$ Jahren ist die Telefonhilfe 147 für Kinder und Jugendliche im ganzen Land rund um die Uhr die Antwort auf diese Fragen. Für den Betrieb dieser spezifischen Dienstleistung unter Aufsicht des Bundes (Zentralstelle für Familienfragen des Bundesamtes für Sozialversicherung) zeichnet die Stiftung pro juventute verantwortlich. Speziell ausgebildete und qualifizierte Telefonberaterinnen/-berater mit Erfahrung in der praktischen Arbeit mit Kindern und Jugendlichen nehmen sich derer an. Sie haben eine ruhige, vertrauensvolle Stimme, sind kommunikativ und können situationsbezogen reagieren. Sie respektieren die durch den Anrufenden als Problem beschriebene Situation. Im interaktiven Gespräch helfen sie den Anrufenden, deren Situation zu verstehen und zu reflektieren. Aus einem lösungsorientierten Beratungsgespräch heraus gewinnen die Anrufenden so Sicherheit und schöpfen Mut. Durch das Stellen der richtigen Fragen werden die bei den Anrufenden vorhandenen Kräfte und Ressourcen aktiviert und gestärkt. Die Telefonberaterinnen/-berater ihrerseits werden in ihrer täglichen und nächtlichen Arbeit begleitet und erhalten Supervision und fortwährende Weiterbildung.

Ob kleine oder grosse Sorgen und Nöte - es ist zumindest nicht an uns Erwachsenen, zu urteilen. Ein Kind, das keinen Gesprächspartner hat. Ein Kind, das sich nicht jederzeit vertrauensvoll an jemanden wenden kann. Ein Kind, das nicht Kind sein kann, wie es dazu das ver-

\section{Die Telefonhilfe 147}

Die Telefonhilfe 147 - das ist das professionelle Beratungsangebot der Stiftung pro juventute, das immer und überall für alle Kinder und Jugendlichen im ganzen Land da ist. Es ist eine sinnvolle Ergänzung zum bestehenden Beratungsnetz. Es macht den Anrufenden Mut und gibt ihnen (wieder) Vertrauen. Mit verschiedenen handlichen Produkten wird eine breitere Bekanntmachung angestrebt. Die Finanzierung der Telefonhilfe 147 ist noch nicht gedeckt!

bürgte Recht hat. Diesem Kind fehlt Grundlegendes, das es für die Entwicklung seiner eigenen Persönlichkeit benötigt. Hier setzt die Telefonhilfe 147 mit ihrer Hilfe und Beratung am Telefon an. Dass diese Nummer im Jahr 2001 bereits 74000 mal angewählt worden ist, zeigt das grosse Bedürfnis nach kompetenter Beratung und Unterstützung.

Das zentrale Phänomen der Telefonberatung ist, dass die Anrufenden häufig mehrere Anläufe benötigen, bis ein Beratungsgespräch effektiv zustande kommt. Es gibt stille, lauschende Anrufe. Es kommt auch vor, dass der Hörer gleich wieder aufgelegt wird. Bei rund $1 / 6$ der eingehenden Anrufe aber wird an weiterhelfende Institutionen verwiesen (Triage). Manchmal geben die Anrufenden später Rückmeldungen zum Erfolg der Beratung. Ansonsten ist aber aufgrund des passiven Beratungssettings und aufgrund der Anonymität ein «Erfolg» der Beratungstätigkeit kaum zu messen. Zu den Anrufen werden einfache standardisierte statistische Daten erfasst. Diese dienen als Grundlage für die Qualitätssicherung und ständige Weiterentwicklung des Angebotes Telefonhilfe 147.

Die Beraterinnen und Berater der Telefonhilfe 147 kennen das bestehende Beratungsnetz und Hilfsangebot im Bereich des Kinderschutzes. Sie verfügen über alle notwendigen Adressen und Kontakte von den vielfältigen Beratungsangeboten, Kriseninterventionsstellen und Kinder- 
schutzgruppen, an welche die Anrufenden vermittelt werden können. Die Beraterinnen und Berater können gegebenenfalls auch - aber nur mit dem expliziten Einverständnis der Anrufenden! - selber Kontakt zu Beratungsstellen oder anderen Vertrauenspersonen der Kinder und Jugendlichen aufnehmen.

Die Mehrheit der Anrufenden sind weiblichen Geschlechts (gut doppelt so viele wie Buben und männliche Jugendliche). Es zeigt sich, dass Mädchen (und Frauen) eher und schneller bereit sind, über Gefühle und Emotionen zu sprechen. Dass sie bereit sind, über sich zu reden. $\mathrm{Zu}$ sagen: Ich habe ein Problem, ich weiss nicht mehr weiter, ich brauche Hilfe. - Und sie holen sich diese Hilfe.

Die Mehrheit der Anrufenden befindet sich im langen, schwierigen und bewegenden Prozess des Erwachsenwerdens mit einem Durchschnittsalter von 12 bis 16 Jahren. Es werden viele Fragen zum Aussehen und zum Körper gestellt (Bin ich normal?). Es geht um die erwachende Sexualität. Um Gefühle, Liebe und erste sexuelle Erfahrungen. Es geht um den Kontakt zu und unter Gleichaltrigen: Ausgeschlossen und verstossen sein, fertig gemacht werden und mehr. Auch Gewalt ist ein Thema, ob im Alltag, in der Schule oder daheim. Die Anrufenden haben Probleme daheim, mit den Geschwistern, mit den Eltern (Ich hau' ab!).

Ein Phänomen ist, dass viele Kinder und Jugendliche «den Blues» haben. Sie suchen den Sinn des Lebens. Sie wissen nicht, welche Ziele sie anstreben möchten. Es fehlt ihnen eine Orientierung. Auch das sinnlich-emotionale Erleben ist keine Selbstverständlichkeit mehr. Neben Ersatzempfindungen zum Beispiel durch Drogen(-erlebnisse) können auch Essstörungen und aktive Selbstzerstümmelung die Folge sein («damit ich mich wieder spüren kann!»).
Nicht jedes Kind schafft es auf Anhieb, den Telefonhörer in die Hand zu nehmen und direkt zu fragen oder zu sagen, was es mitzuteilen hat (Wie sag ich's?). Die Kinder und Jugendlichen «spielen» mit dem Telefon: Sie rufen mehrfach an, legen wieder auf, sie lauschen, verstellen die Stimme, erzählen in der Drittperson, bis das Eis gebrochen und das Vertrauen da ist. Auch dann braucht es noch viel Erfahrung, Geduld und Geschick, um die dahinterliegende Problematik $\mathrm{zu}$ erkennen und dem anrufenden Kind oder Jugendlichen wieder Mut machen zu können.

Auch bei Kindern und Jugendlichen früher wenig bekannte Phänomene wie Depressionen und Suizidalität haben zugenommen. Das Telefon kann hier das Medium sein, das hilft, einen Ausweg und neue Perspektiven zu finden.

Die für den Betrieb der Telefonhilfe 147 benötigten Finanzen sind bis heute nicht gedeckt! Der fehlende Betrag (über 30\%) muss durch die den Dienst leistenden Organisationen selber beigesteuert werden. Es sind bei weitem noch nicht alle Kantone bereit, ihren Teil der sozialen Verantwortung durch die Mitfinanzierung dieser Lücke im sozialen Netz zu tragen.

Die Telefonhilfe 147 ist darauf angewiesen, dass möglichst viele Botschafter und Multiplikatorinnen und Multiplikatoren die Dienstleistung und deren Ziel weitertragen und kommunizieren. Insbesondere dort, wo sich die Zielgruppe der Kinder und Jugendlichen aufhält.

An die Schweizerischen (Kinder-) Ärzte gelangen wir mit dem Appell, die kindergerechten Werbematerialien (Kreditkärtchen, Flyers, Kleber, Zeltli) zu verteilen und in ihren Praxen aufzulegen. Das Sekretariat der Telefonhilfe 147 in Zürich verschickt auf Anfrage gerne die entsprechenden Materialien: pro juventute, Telefonhilfe 147, Postfach, 8032 Zürich, Tel. 0125677 54, E-Mail: katalin.kunz@projuventute.ch,www.147.ch. 